ARTIGO ORIGINAL

\title{
Banco de sementes do solo sob plantios de Tachigali vulgaris (L.G.Silva \& H.C.Lima) em diferentes espaçamentos na região leste do Estado do Pará, Amazônia
}

\author{
Soil seed bank under Tachigali vulgaris (L.G.Silva \& H.C.Lima) plantations in \\ different spacing in the eastern region of the State of Pará, Amazon
}

\author{
Tainah Silva Narducci $^{1}$ (D), Jorge Alberto Gazel Yared ${ }^{2}$ (D), Silvio Brienza Júnior ${ }^{3}$ (1) \\ ${ }^{1}$ Universidade Federal do Pará - UFPA, Belém, PA, Brasil \\ ${ }^{2}$ Empresa Brasileira de Pesquisa Agropecuária - Embrapa Amapá, Macapá, AP, Brasil \\ ${ }^{3}$ Empresa Brasileira de Pesquisa Agropecuária - Embrapa Amazônia Oriental, Belém, PA, Brasil
}

Como citar: Narducci, T. S., Yared, J. A. G., \& Brienza Júnior, S. (2021). Banco de sementes do solo sob plantios de Tachigali vulgaris (L.G.Silva \& H.C.Lima) em diferentes espaçamentos na região leste do Estado do Pará, Amazônia. Scientia Forestalis, 49(132), e3719. https://doi.org/10.18671/scifor.v49n132.11

\begin{abstract}
Resumo
O objetivo neste trabalho foi investigar a composição florística, riqueza, diversidade, equabilidade e similaridade do banco de sementes do solo de um plantio de Tachigali vulgaris L.G.Silva \& H.C.Lima em diferentes espaçamentos ( $4 \mathrm{~m} \times 2 \mathrm{~m}, 4 \mathrm{~m} \times 3 \mathrm{~m}$ e $4 \mathrm{~m} \times 4 \mathrm{~m}$ ) com aproximadamente sete anos e meio de idade, localizado na Fazenda Gênesis em Dom Eliseu (PA). As amostras do banco de sementes foram coletadas em parcelas de $30 \mathrm{~m} \times 50 \mathrm{~m}$ com quatro repetições para cada espaçamento, sendo sorteados aleatoriamente oito pontos em cada parcela para coleta de solo na profundidade de $5 \mathrm{~cm}$. O solo coletado dos oitos pontos foi misturado, e a partir da mistura foram retiradas quatro amostras compostas para cada parcela, totalizando 16 amostras para cada espaçamento. As amostras de solo contendo as sementes foram colocadas para emergência das plântulas na casa de vegetação da Embrapa Amazônia Oriental. As plântulas foram contadas e identificadas mensalmente durante quatro meses. As herbáceas se destacaram tanto em presença como em abundância das espécies nos três espaçamentos. No espaçamento $4 \mathrm{~m} \times 3 \mathrm{~m}$ foi observada maior estimativa de riqueza Jackknife I. Os três espaçamentos apresentaram baixa diversidade e equabilidade de espécies e alta similaridade florística. O banco de sementes no solo apresentou as mesmas características na composição florística, presença e abundância de herbáceas, diversidade, equabilidade e similaridade nos três espaçamentos analisados. Qualquer um dos três espaçamentos de Tachigali vulgaris pode ser utilizado para o plantio desta espécie com o intuito de recuperação de áreas.
\end{abstract}

Palavras-chave: Composição florística; Diversidade; Equabilidade.

\footnotetext{
Abstract
}

The objective of this study was to investigate the floristic composition, richness, diversity, evenness and similarity of the soil seed bank of a plantation of Tachigali vulgaris L.G.Silva \& H.C.Lima in different spacings ( $4 \mathrm{~m} \times 2 \mathrm{~m}, 4 \mathrm{~m} \times 3 \mathrm{~m}$ and $4 \mathrm{~m} \times 4 \mathrm{~m})$ at approximately seven and half years of age, located in the Genesis Farm in Dom Eliseu (PA). Seed bank samples were collected in $30 \mathrm{~m} \times 50 \mathrm{~m}$ plots with four replications for each spacing, eight points were randomly drawn in each plot to collect soil at a depth of $5 \mathrm{~cm}$. The soil collected from the eight points was mixed, and from the mixture, four composite samples were taken for each plot, totaling 16

Fonte de financiamento: Nenhuma.

Conflito de interesse: Nada a declarar.

Autor correspondente: tainahnarducci@hotmail.com

Recebido: 23 maio 2021.

Aceito: 6 agosto 2021

Editor: Mauro Valdir Schumacher.

(c) (i) Este é um artigo publicado em acesso aberto (Open Access) sob a licença Creative Commons Attribution, que permite uso, distribuição e reprodução em qualquer meio, sem restrições desde que o trabalho original seja corretamente citado. 
samples for each spacing. Soil samples containing the seeds were placed for seedling emergence in the greenhouse of Embrapa Amazônia Oriental. Seedlings were counted and identified monthly for four months. Herbaceous plants stood out both in presence and abundance of species in the three spacings. In the $4 \mathrm{~m} \times 3 \mathrm{~m}$ spacing the highest estimate of Jackknife I richness was observed. The three spacings showed low species diversity and evenness and high floristic similarity. The seed bank in the soil presented the same characteristics in floristic composition, presence and abundance of herbaceous plants, diversity, evenness and similarity in the three spacings analyzed. Any of the three spacings of Tachigali vulgaris can be used in planting this species for area recovery.

Keywords: Floristic composition; Diversity; Equability.

\section{INTRODUÇÃO}

O desmatamento na Amazônia gera impactos socioambientais que envolvem desde queimadas para expansão agrária, mudanças no uso da terra e concentração latifundiária (Domingues \& Bermann, 2012). Em adição a esse fato, a exploração madeireira promove alteração na estrutura natural dos ecossistemas além do empobrecimento de espécies de valor econômico das florestas remanescentes (Araujo et al., 2001). As florestas degradadas na Amazônia Legal somaram $399 \mathrm{~km}^{2}$, em dezembro de 2020, o que correspondeu a um aumento de $5 \%$ em relação aos $380 \mathrm{~km}^{2}$ de degradação detectados em dezembro de 2019 (Fonseca et al., 2020).

A intensidade e o tipo desse distúrbio dependem da natureza do processo de usos da terra que ocorrem nas diferentes regiões alteradas, o que exerce influência no padrão de recuperação natural do ecossistema (Araujo et al., 2001). Áreas utilizadas com tecnologias apropriadas mantêm-se produtivas por muito tempo enquanto grande parte das áreas alteradas são deixadas em recuperação por um processo natural em longo tempo.

O tema recuperação de áreas degradadas e/ou alteradas tem despertado a atenção do meio científico (Calegari et al., 2013; Campos Filho \& Sartorelli, 2016; Zappi et al., 2018). Um dos principais desafios da pesquisa é desenvolver e apresentar inovações e alternativas tecnológicas para a recuperação das áreas degradadas de forma mais célere, seja para a recuperação ambiental (Aumond, 2019) ou para a incorporação dessas áreas em processos produtivos visando à produção de bens de valor econômico (Rodrigues et al., 2020). Áreas que passaram por um processo acentuado de degradação, com intensa erosão e que houve a retirada do solo, devem ser recuperadas primeiramente com plantio de pioneiras agressivas, de preferência as leguminosas, após tal procedimento, com o solo recuperado e protegido, são introduzidas as espécies não pioneiras (Macedo, 1993).

Na Amazônia, pesquisas apontaram o uso do plantio florestal com Tachigali vulgaris L.G.Silva \& H.C.Lima (Fabaceae), leguminosa arbórea, que desempenha papel fundamental na recuperação de áreas degradadas (Mochiutti et al., 1999; Carvalho, 2005; Freitas et al., 2012). Essa espécie que pertence ao grupo ecológico das pioneiras (Amaral et al., 2009), promove diversas condições benéficas para o desenvolvimento de outras pioneiras, as quais são importantes por favorecer maior diversidade biológica na sucessão da seleção natural (Souza et al., 2004). Em geral, por apresentarem rápido crescimento, as espécies pioneiras acabam propiciando o sombreamento necessário para o desenvolvimento e sobrevivência de espécies secundárias tardias e clímax (Martins, 2013).

Outras características promissoras para o uso da Tachigali vulgaris na recuperação de áreas são as evidências existentes de que a espécie é amplamente procurada por polinizadores (Sousa et al., 2016) e produz muita liteira, a qual proporciona melhoria na atividade biológica em solos altamente intemperizados e na formação de horizontes orgânicos no solo (Mochiutti et al., 2006). Certamente, o plantio de árvores apropriadas pode funcionar positivamente como mecanismo para acelerar o processo de recuperação de áreas alteradas. 
Em outra dimensão, o banco de sementes do solo é também um fator importante que pode favorecer a regeneração natural das florestas, embora a sua presença na agricultura esteja muito mais associada à competição com as plantas cultivadas (Costa et al., 2013a). O banco de sementes do solo está associado ao estabelecimento de espécies de diferentes grupos ecológicos com finalidades específicas na recolonização natural de áreas perturbadas (Baider et al., 1999). A duração e o grau do distúrbio do solo têm influência direta no banco de sementes que, em casos extremos, pode ser perdido, dificultando a regeneração natural do local (Machado et al., 2013; Martins, 2013).

Fatores ambientais são importantes na dinâmica do banco de sementes do solo (Oliveira Júnior et al., 2011). Entre diversos fatores, a presença de luz e a matéria orgânica pode influenciar a qualidade e quantidade do banco de sementes no solo. Brandão et al. (2016) mencionam que um dossel mais aberto da floresta secundária pode facilitar a dispersão e a incorporação de sementes no solo. Por outro lado, esses mesmos autores concluíram, em seus estudos, que a densa camada de serapilheira impediu a chegada de propágulos e a germinação das poucas sementes presentes no solo, inibindo a sucessão secundária.

Em cultivos agrícolas, o banco de sementes do solo pode ser afetado pelas práticas de manejo que são utilizadas como estratégias no controle da competição com as plantas cultivadas (Favreto \& Medeiros, 2006; Oliveira Júnior. et al., 2011; Costa et al., 2013a; Bloemer et al., 2017). A densidade de plantio nos cultivos agrícolas pode afetar o banco de sementes do solo, conforme demonstrado no trabalho de Bloemer et al. (2017) sobre os estudos de arranjo populacional na cultura de milho e o manejo de plantas daninhas. Em um estudo de espaçamento de plantio com plantas de cafeeiro, Carvalho et al. (2015) concluíram que há predominância de determinadas espécies independentemente do arranjo espacial de plantio. Na literatura especializada em ciência florestal, no Brasil, não foram encontrados trabalhos versando sobre a avaliação do banco de sementes do solo em plantio de espécies florestais em diferentes espaçamentos.

Na Amazônia, estudos têm sido realizados sobre bancos de sementes em diferentes situações ambientais com o propósito de identificar o seu funcionamento (Monaco et al., 2003; Bordon, 2012; Lau \& Jardim, 2014; Rayol \& Alvino-Rayol, 2018; Alvino-Rayol \& Rayol, 2019). O conhecimento sobre a regeneração natural e a dinâmica do banco de sementes do solo é um procedimento importante para avaliar o potencial de recuperação de áreas degradadas (Calegari et al., 2013).

Neste trabalho, o objetivo foi avaliar o efeito de diferentes espaçamentos de plantio de Tachigali vulgaris na composição florística, riqueza, diversidade, equabilidade e similaridade do banco de sementes do solo com o intuito de verificar se há um possível espaçamento que possa ser utilizado para recuperação de áreas, além de contribuir no aperfeiçoamento de técnicas economicamente mais viáveis.

\section{MATERIAL E MÉTODOS}

O estudo foi realizado na Fazenda Gênesis, no município de Dom Eliseu (PA), latitude $04^{\circ} 33^{\prime} 10^{\prime \prime} \mathrm{S}$, longitude $47^{\circ} 49^{\prime} 0^{\prime \prime} \mathrm{W}$ e altitude de $180 \mathrm{~m}$. As características climáticas do município são: umidade relativa do ar por volta de $85 \%$; regime pluviométrico geralmente está entre $2.250 \mathrm{~mm}$ e $2.500 \mathrm{~mm}$; as chuvas são regulares, mas não se distribuem igualmente durante o ano, apresentam maior concentração de janeiro a junho; o clima é caracterizado como mesotérmico úmido; e a temperatura média anual é em torno de $25^{\circ} \mathrm{C}$ (Pará, 2013).

O solo onde foi realizado a coleta é classificado como Latossolo Amarelo Distrófico A, moderado textura muito argilosa. A cobertura vegetal anterior ao plantio de Tachigali vulgaris era Floresta Tropical Úmida, que foi intensamente explorada, e em outubro de 2005 houve a supressão total da vegetação (Sousa, 2011).

O plantio das mudas de Tachigali vulgaris (tachi-branco) foi realizado em fevereiro de 2006 e distribuídas em pequenos blocos constituídos pelos espaçamentos $4 \mathrm{~m} \times 2 \mathrm{~m} ; 4 \mathrm{~m} \times 3 \mathrm{~m}$; e, 
$4 \mathrm{~m} \times 4 \mathrm{~m}$. A última limpeza na área do plantio foi feita no início de 2010 , e as coletas das amostras do banco de sementes do solo foram realizadas em agosto de 2013, quando o plantio das árvores tinha, aproximadamente, sete anos e meio de idade.

As amostras do solo foram coletadas em parcelas com dimensões de $30 \mathrm{~m} \times 50 \mathrm{~m}$ com quatro repetições para cada espaçamento. Em cada parcela, foram sorteados aleatoriamente oito pontos de coleta do solo na profundidade de $5 \mathrm{~cm}$, os quais foram misturados, e a partir dessa mistura foram retiradas quatro amostras compostas, ou seja, totalizando 16 amostras para cada espaçamento. O solo foi coletado com auxílio de espátula e gabarito $(25 \mathrm{~cm} \times 25 \mathrm{~cm})$, desprezando-se o excesso de liteira.

As 48 amostras foram transportadas em sacos de plástico (transparentes e identificados) para casa de vegetação da Embrapa Amazônia Oriental - Belém/PA. As amostras foram postas em bandejas de plástico $(27 \mathrm{~cm} \times 41 \mathrm{~cm} \times 7 \mathrm{~cm})$, as quais foram distribuídas aleatoriamente e regadas diariamente. A contagem e identificação das plântulas que emergiram foram realizadas mensalmente, durante quatro meses. Após cada identificação, as plântulas eram retiradas das bandejas. Com dois meses de monitoramento, o solo foi revolvido para dar oportunidade de germinação para todas as sementes.

As plântulas do banco de sementes foram identificadas por um parabotânico da Embrapa Amazônia Oriental. Os nomes científicos das espécies, suas famílias e seus respectivos autores foram checados nos sites do Tropicos (2017) e da Flora do Brasil 2020 (2017). As espécies foram classificadas por forma de vida através de consultas a especialistas e na plataforma da Flora do Brasil 2020 (2017).

O perfil vertical da abundância relativa das espécies por formas de vida presentes no banco de sementes do solo nos três espaçamentos de plantio foi representado por imagem utilizando o programa AUTOCAD. Foi calculada a abundância ou densidade relativa das espécies que se refere a densidade de uma espécie dividida pela somatória da densidade total das espécies (Villarreal et al., 2004).

Para a comparação da riqueza de espécies entre os três espaçamentos, curvas de acúmulo de espécies de rarefação e extrapolação baseadas no tamanho da amostra (Gotelli \& Colwell, 2011a) foram construídas usando a função iNEXT no pacote iNEXT (Hsieh et al., 2020) e números de Hill para interpolação e extrapolação (Chao et al., 2014). Desse modo, foi criada uma curva de rarefação com 999 permutações. Para verificar se a riqueza observada está próxima da riqueza estimada nos três espaçamentos, foi utilizado o estimador Jackknife I com 999 permutações (Magurran, 2004; Gotelli \& Ellison, 2011b).

Curvas de abundâncias foram elaboradas para os três espaçamentos. Na representação gráfica, as classificações das abundâncias das espécies são apresentadas ao longo do eixo horizontal em ordem decrescente de abundância relativa, o que facilita as comparações de comunidades (Magurran, 2004; Gotelli \& Ellison, 2011b).

Por fim, os índices de diversidade de Shannon e de equabilidade de Pielou e de similaridade de Sørensen foram calculados conforme metodologia adotada por Villarreal et al. (2004).

\section{RESULTADOS E DISCUSSÃO}

Da análise do banco de sementes do solo foi encontrado um total de 6395 plântulas, distribuídas nos seguintes espaçamentos: 2594 plântulas no espaçamento $4 \mathrm{~m} \times 2$ m; 2079 plântulas no espaçamento $4 \mathrm{~m} \times 3 \mathrm{~m}$; e, 1722 plântulas no espaçamento $4 \mathrm{~m} \times 4 \mathrm{~m}$.

No banco de sementes foi observado um total de 27 famílias, 46 gêneros e 57 espécies (Tabela 1). No espaçamento $4 \mathrm{~m} \times 2 \mathrm{~m}$ foram encontradas 41 espécies pertencentes a 23 famílias, enquanto no espaçamento $4 \mathrm{~m} \times 3 \mathrm{~m}$ foram observadas 45 espécies e 25 famílias e no espaçamento $4 \mathrm{~m} \times 4 \mathrm{~m}$ foram identificadas 32 espécies e 19 famílias. 
Tabela 1. Relação das espécies e de formas de vida do banco de sementes do solo no plantio de Tachigali vulgaris plantados em diferentes espaçamentos, Fazenda Gênesis, Dom Eliseu (PA).

\begin{tabular}{|c|c|c|c|c|}
\hline \multirow{2}{*}{ FAMÍLIA/Espécie } & \multirow{2}{*}{ Forma de vida } & \multicolumn{3}{|c|}{ Espaçamentos $(\mathrm{m} \times \mathrm{m})$} \\
\hline & & $4 \times 2$ & $4 \times 3$ & $4 \times 4$ \\
\hline \multicolumn{5}{|l|}{ ASTERACEAE } \\
\hline Bidens cynapiifolia Kunth & Erva & $x$ & $x$ & $x$ \\
\hline Emilia sonchifolia (L.) DC. & Erva & $x$ & $x$ & $x$ \\
\hline Erechtites hieracifolius (L.) Raf. ex DC. & Erva & $x$ & $x$ & \\
\hline Porophyllum ruderale (Jacq.) Cass. & Erva & $x$ & & \\
\hline Rolandra fruticosa (L.) Kuntze & Arbusto & & & $x$ \\
\hline Tilesia baccata (L.f.) Pruski & Arbusto & & $x$ & \\
\hline \multicolumn{5}{|l|}{ CANNABACEAE } \\
\hline \multicolumn{4}{|l|}{ CLEOMACEAE } & $x$ \\
\hline \multicolumn{4}{|l|}{ CYPERACEAE } & \\
\hline Cyperus laxus Lam. & Erva & $x$ & $x$ & $x$ \\
\hline Fimbristylis miliacea (L.) Vahl & Erva & $x$ & $x$ & $x$ \\
\hline Rhynchospora ciliata (Vahl) Kük. & Erva & $x$ & $x$ & $x$ \\
\hline $\begin{array}{l}\text { Scleria gaertneri Raddi } \\
\text { EUPHORBIACEAE }\end{array}$ & Erva & $x$ & $x$ & $x$ \\
\hline Acalypha arvensis Poepp. & Erva & $x$ & $x$ & $x$ \\
\hline Astraea lobata (L.) Klotzsch & Erva & $x$ & & \\
\hline Croton grandivelus Baill. & Arbusto & & $x$ & \\
\hline Croton matourensis Aubl. & Árvore & $x$ & $x$ & $x$ \\
\hline $\begin{array}{l}\text { Croton trinitatis Millsp. } \\
\text { FABACEAE }\end{array}$ & Erva & $x$ & & \\
\hline Chamaecrista riparia (Kunth) Britton & Erva & $x$ & $x$ & $x$ \\
\hline Desmodium barbatum (L.) Benth. & Erva & $x$ & $x$ & $x$ \\
\hline Helicotropis linearis (Kunth) A. Delgado & Liana & & & $x$ \\
\hline Mimosa dormiens Humb. \& Bonpl. ex Willd. & Erva & $x$ & & \\
\hline $\begin{array}{l}\text { Mimosa pudica } \mathrm{L} \text {. } \\
\text { GENTIANACEAE }\end{array}$ & Erva & $x$ & $x$ & $x$ \\
\hline \multicolumn{4}{|l|}{ HYPERICACEAE } & \\
\hline \multicolumn{5}{|l|}{ LACISTEMATACEAE } \\
\hline \multicolumn{4}{|l|}{ LAMIACEAE } & $x$ \\
\hline $\begin{array}{l}\text { Hyptis atrorubens Poit. } \\
\text { LINDERNIACEAE }\end{array}$ & Erva & $x$ & $x$ & $x$ \\
\hline $\begin{array}{l}\text { Lindernia crustacea (L.) F.Muell. } \\
\text { Lindernia diffusa (L.) Wettst. } \\
\text { LOGANIACEAE }\end{array}$ & $\begin{array}{l}\text { Erva } \\
\text { Erva }\end{array}$ & $x$ & $\begin{array}{l}x \\
x\end{array}$ & $x$ \\
\hline $\begin{array}{l}\text { Spigelia anthelmia L. } \\
\text { LYGODIACEAE }\end{array}$ & Erva & $x$ & $x$ & \\
\hline $\begin{array}{l}\text { Lygodium venustum SW. } \\
\text { MALVACEAE }\end{array}$ & Erva & $x$ & & \\
\hline Melochia villosa (Mill.) Fawc. \& Rendle var. villosa & Arbusto & $x$ & & \\
\hline $\begin{array}{l}\text { Urena lobata } \mathrm{L} . \\
\text { OCHNACEAE }\end{array}$ & Arbusto & & $x$ & \\
\hline $\begin{array}{l}\text { Sauvagesia erecta L. } \\
\text { PASSIFLORACEAE }\end{array}$ & Erva & $x$ & $x$ & \\
\hline $\begin{array}{l}\text { Passiflora poeppigii Mast. } \\
\text { PHYLLANTHACEAE }\end{array}$ & Liana & $x$ & $x$ & $x$ \\
\hline
\end{tabular}


Tabela 1. Relação das espécies e de formas de vida do banco de sementes do solo no plantio de Tachigali vulgaris plantados em diferentes espaçamentos, Fazenda Gênesis, Dom Eliseu (PA).

\begin{tabular}{|c|c|c|c|c|}
\hline \multirow{2}{*}{ FAMÍLIA/Espécie } & \multirow{2}{*}{ Forma de vida } & \multicolumn{3}{|c|}{ Espaçamentos $(\mathrm{m} \times \mathrm{m})$} \\
\hline & & $4 \times 2$ & $4 \times 3$ & $4 \times 4$ \\
\hline Phyllanthus niruri L. & Erva & $x$ & $x$ & $x$ \\
\hline Phyllanthus orbiculatus Rich. & Erva & & $x$ & \\
\hline $\begin{array}{l}\text { Phyllanthus urinaria } \mathrm{L} \text {. } \\
\text { PHYTOLACCACEAE }\end{array}$ & Erva & $x$ & & $x$ \\
\hline $\begin{array}{l}\text { Phytolacca rivinoides Kunth \& Bouché } \\
\text { PLANTAGINACEAE }\end{array}$ & Erva & $x$ & $x$ & $x$ \\
\hline $\begin{array}{l}\text { Scoparia dulcis L. } \\
\text { POACEAE }\end{array}$ & Erva & $x$ & $x$ & $x$ \\
\hline Cynodon dactylon (L.) Pers. & Erva & $x$ & & \\
\hline Eleusine indica (L.) Gaertn. & Erva & & $x$ & \\
\hline Paspalum conjugatum P.J. Bergius & Erva & $x$ & $x$ & $x$ \\
\hline Rugoloa pilosa (SW.) Zuloaga & Erva & $x$ & $x$ & $x$ \\
\hline $\begin{array}{c}\text { Steinchisma laxum (SW.) Zuloaga } \\
\text { POLYGALACEAE }\end{array}$ & Erva & $x$ & $x$ & $x$ \\
\hline $\begin{array}{c}\text { Asemeia violacea (Aubl.) J.F.B. Pastore \& J.R. Abbott } \\
\text { RUBIACEAE }\end{array}$ & Erva & & $x$ & \\
\hline Borreria latifolia (Aubl.) K.Schum. & Erva & $x$ & $x$ & $x$ \\
\hline Borreria ocymifolia (Roem. \& Schult.) Bacigalupo \& E.L. Cabral & Erva & & $x$ & \\
\hline Borreria Suaveolens G. Mey. & Erva & & $x$ & \\
\hline Borreria verticillata (L.) G. Mey & Erva & $x$ & $x$ & $x$ \\
\hline $\begin{array}{c}\text { Galianthe laxa (Cham. \& Schldl.) E.L. Cabral } \\
\text { RUTACEAE }\end{array}$ & Erva & & $x$ & \\
\hline $\begin{array}{l}\text { Zanthoxylum rhoifolium Lam. } \\
\text { SALICACEAE }\end{array}$ & Árvore & $x$ & $x$ & $x$ \\
\hline $\begin{array}{l}\text { Casearia arborea (Rich.) Urb. } \\
\text { SOLANACEAE }\end{array}$ & Árvore & & $\mathrm{X}$ & \\
\hline Solanum caavurana Vell. & Arbusto & & $\mathrm{X}$ & \\
\hline Solanum crinitum Lam. & Arbusto & $x$ & & \\
\hline $\begin{array}{l}\text { Solanum subinerme Jacq. } \\
\text { URTICACEAE }\end{array}$ & Arbusto & $x$ & $x$ & $x$ \\
\hline $\begin{array}{c}\text { Cecropia obtusa Trécul } \\
\text { VERBENACEAE }\end{array}$ & Árvore & $x$ & $x$ & $x$ \\
\hline Stachytarpheta cayennensis (Rich.) Vahl & Erva & $x$ & $x$ & $x$ \\
\hline
\end{tabular}

As famílias que apresentaram maior riqueza em espécies na área total avaliada foram: Asteraceae com 6 espécies e Euphorbiaceae, Fabaceae, Poaceae e Rubiaceae com 5 espécies cada.

Asteraceae apresentou maior riqueza em espécies, cujas características principais são dadas pelas formas de vida como as ervas, arbustos, subarbustos e, eventualmente, árvores ou lianas (Roque \& Bautista, 2008). A riqueza de espécies pertencentes a essa família também foi constatada em banco de sementes de outras áreas que sofreram algum tipo de impacto (Martins et al., 2008; Miranda Neto et al., 2010). Além da Asteraceae, seguiu-se a família Poaceae como uma das mais ricas neste presente trabalho; essas duas famílias mostraram maior número de espécies em plantios de Pinus spp. e Eucalyptus spp. em outras regiões do país (Gonçalves et al., 2008). De forma similar, Lau \& Jardim (2014) também encontraram que as famílias mais representativas foram Poaceae, Asteraceae e Cyperaceae, com quatro espécies cada, ainda que os estudos tenham ocorrido em áreas de florestas de várzea, na Amazônia.

Quanto à forma de vida, o banco de sementes do solo do plantio de Tachigali vulgaris, na área total de estudo apresentou $70,2 \%$ de ervas, $14,0 \%$ de arbustos, $12,3 \%$ de árvores e 3,5\% de lianas. O menor percentual de presença de cipós 
na área parece um aspecto favorável ao manejo das espécies arbóreas plantadas ou da regeneração natural do sub-bosque, por evitar maior concorrência pelos fatores de produção ao desenvolvimento dessas espécies.

A abundância relativa das espécies por formas de vida encontradas no banco de sementes do solo, nos diferentes espaçamentos, é ilustrada na Figura 1, destacando-se a abundância de ervas, com valores acima de $90 \%$ em cada espaçamento comparativamente às demais formas de vida.

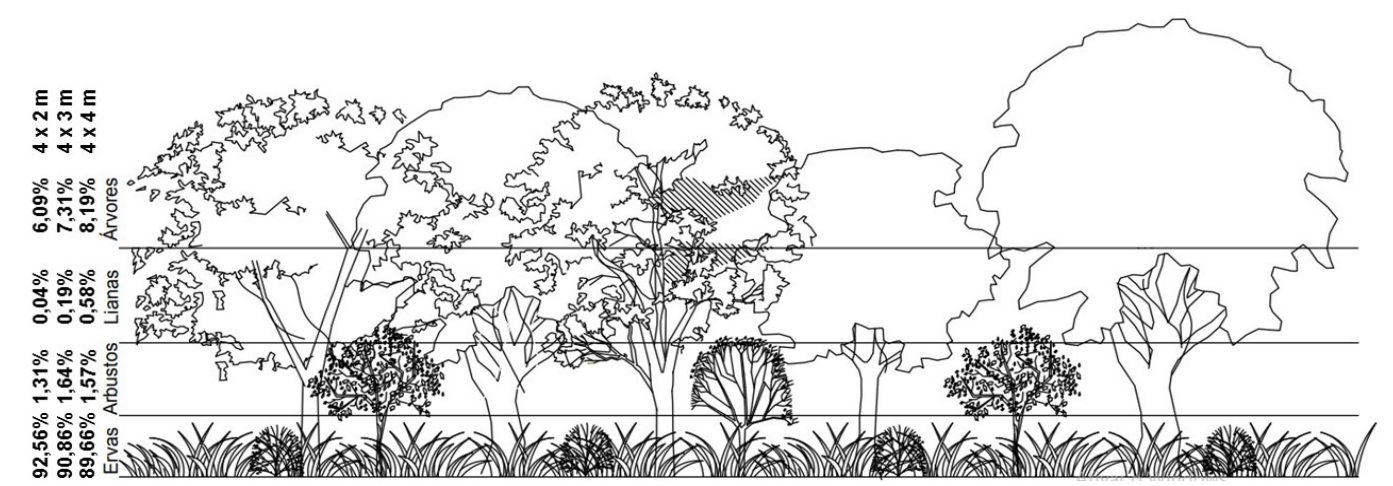

Figura 1. Abundância relativa das espécies por formas de vida do banco de sementes do solo dos três espaçamentos de plantio de Tachigali vulgaris.

As espécies que apresentaram maior densidade relativa na área total estudada foram Cyperus laxus (30,7\%), Rugoloa pilosa (20\%), Paspalum conjugatum (12,8\%) e Borreria verticillata (7,4\%). A soma das espécies com maior densidade relativa representa mais de $60 \%$ de densidade relativa em cada espaçamento de plantio, e todas são classificadas como ervas (Tabela 2).

Tabela 2. Densidade relativa (DR) das espécies com maior percentagem no banco de sementes do solo de Tachigali vulgaris nos três espaçamentos de plantio.

\begin{tabular}{|c|c|c|c|}
\hline & \multicolumn{3}{|c|}{ Densidade Relativa (DR) } \\
\hline & $4 \mathrm{~m} \times 2 \mathrm{~m}(\%)$ & $4 \mathrm{~m} \times 3 \mathrm{~m}(\%)$ & $4 \mathrm{~m} \times 4 \mathrm{~m}(\%)$ \\
\hline Borreria verticillata & 13,3 & - & - \\
\hline Cyperus laxus & 15,2 & 42,2 & 40,2 \\
\hline Paspalum conjugatum & 10,3 & 12,0 & 17,5 \\
\hline Rugoloa pilosa & 30,7 & 14,8 & 10,3 \\
\hline
\end{tabular}

As herbáceas também se destacaram em outros trabalhos, sobre banco de sementes do solo, realizados por diversos autores, sob condições de vegetação natural ou de diferentes espécies plantadas e em diferentes regiões. Destacam-se os resultados encontrados em estudos realizados em um trecho de floresta montana madura circundada por florestas em regeneração (Baider et al., 1999), em um fragmento de floresta estacional decidual ripária próximo aos campos e áreas agrícolas (Araujo et al., 2004), em uma vegetação secundária numa área degradada por mineração (Martins et al., 2008) e em plantios de Eucalyptus grandis W.Hill (Seubert et al., 2016), Pinus spp. e Eucalyptus spp. (Gonçalves et al., 2008). Nesses dois últimos plantios, os autores concluíram que a predominância das espécies herbáceas demostra o estágio inicial de regeneração do sub-bosque.

De acordo com Franco et al. (2012), a prevalência de herbáceas em um banco de sementes de um fragmento florestal pode ser devido à fragmentação deixar as bordas mais vulneráveis às perturbações antrópicas e também quando a área que rodeia esse fragmento apresenta anos de atividades agrícolas. Gonçalves et al. (2008) mencionou que quanto maior a intervenção nos povoamentos, maior número de sementes de espécies herbáceas. Araujo et al. (2004) afirmam que as herbáceas, apesar de serem geralmente consideradas 
como espécies invasoras, agem no primeiro estágio de colonização do ambiente modificado, o que as tornam essenciais no processo de regeneração.

A infestação elevada de herbáceas invasoras, principalmente gramíneas exóticas e trepadeiras agressivas, pode inibir a regeneração natural de espécies arbustivo-arbóreas (Franco et al., 2012), sendo que é recomendado proceder-se a intervenção no controle dessas plantas agressivas estimulando a regeneração natural das arbustivo-arbóreas (Martins, 2013). No trabalho de Costa et al. (2013b), as herbáceas mostraram ser importantes, pois atuam como indicadoras da qualidade do ambiente, devido à capacidade de se adaptarem e se desenvolverem em ambientes modificados.

A riqueza observada se aproxima da riqueza estimada para os três espaçamentos conforme o estimador de Jackknife I. A riqueza observada para o espaçamento $4 \mathrm{~m} \times 2 \mathrm{~m}$ foi de 41 espécies e a estimada 49 7 . Para o espaçamento $4 \mathrm{~m} \times 3 \mathrm{~m}$ a riqueza observada foi de 45 espécies e a estimada 56 \pm 9 . Para o espaçamento $4 \mathrm{~m} \times 4 \mathrm{~m}$, a riqueza observada foi 32 espécies e a riqueza estimada foi $38 \pm 7$ espécies. A riqueza de espécies diferiu entre os três espaçamentos, uma vez que pouco se evidenciou a sobreposição dos intervalos de confiança, e as curvas de rarefação apresentaram assíntotas (Figura 2).

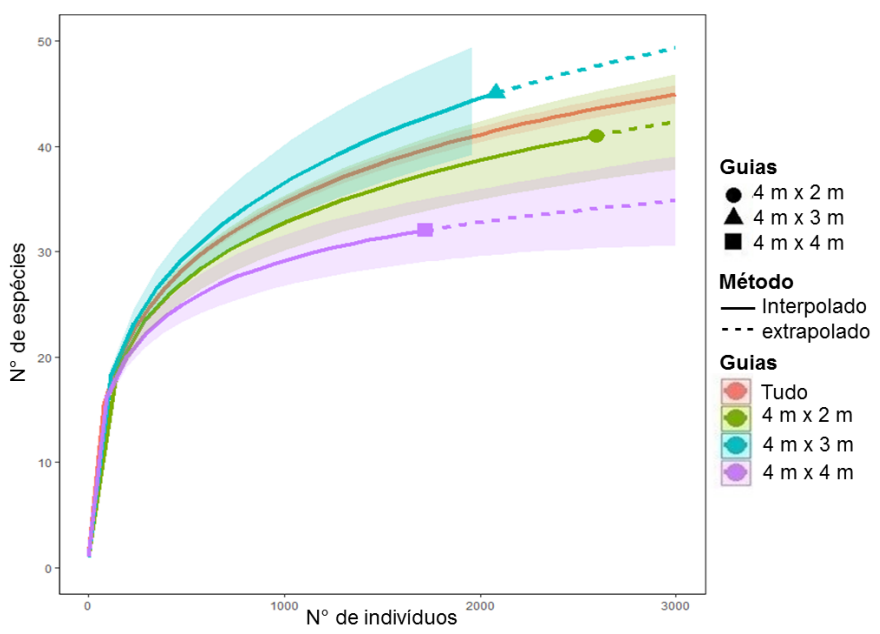

Figura 2. Curva de rarefação nos espaçamentos de plantio de Tachigali vulgaris.

Ao se analisar a relação da abundância relativa com o número de espécies (Figura 3), considerando-se os três espaçamentos, constatou-se a presença de poucas espécies abundantes, sendo que, em sua maioria, a riqueza foi constituída de espécies pouco abundantes ou raras.

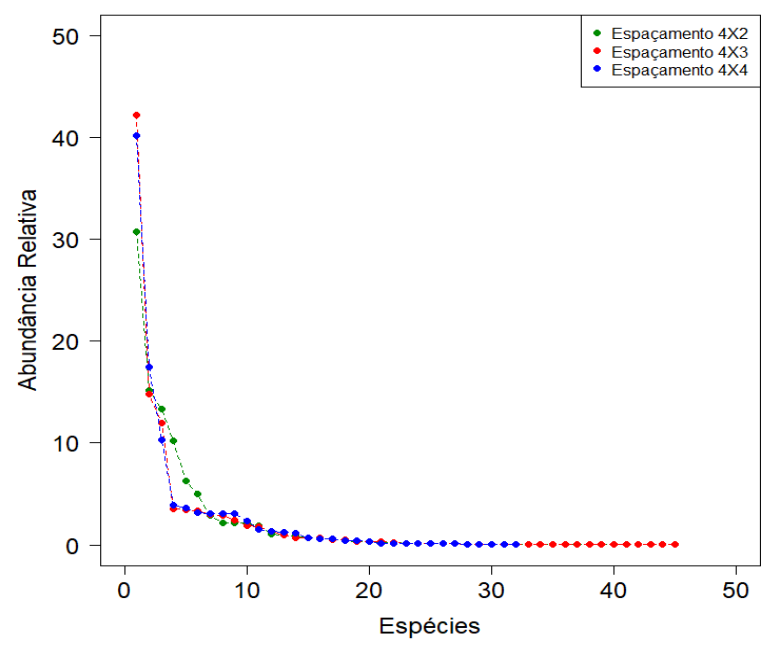

Figura 3. Curvas de abundância relativa nos três espaçamentos de plantio de Tachigali vulgaris. 
Os valores dos índices de diversidade de Shannon variaram de 2,33 a 2,15 (Tabela 3), com uma leve diminuição do menor ( $4 \mathrm{~m} \times 2 \mathrm{~m}$ ) para o maior espaçamento $(4 \mathrm{~m} \times 4 \mathrm{~m})$, sendo que a mesma tendência não ocorreu em relação à equabilidade de Pielou, cujos valores variaram de 0,63 (4 m x $2 \mathrm{~m})$ a 0,57 (4 m x $3 \mathrm{~m})$. O valor baixo do índice de diversidade de Shannon significa que poucas espécies foram responsáveis pela maior proporção de sementes no solo, o que é corroborado na representação gráfica das curvas de abundância relativa dos três espaçamentos de plantio adotados nesta pesquisa (Figura 3).

Tabela 3. Índice de diversidade de Shannon $\left(\mathrm{H}^{\prime}\right)$, de equabilidade de Pielou (J) e de similaridade de Sørensen (ISS) do banco de sementes do solo nos três espaçamentos de plantio.

\begin{tabular}{|c|c|c|c|c|}
\hline & \multirow{2}{*}{$\mathbf{H}^{\prime}$} & \multirow{2}{*}{ J } & \multicolumn{2}{|c|}{ ISS } \\
\hline & & & $4 m \times 2 m$ & $4 \mathrm{~m} \times 3 \mathrm{~m}$ \\
\hline $4 m \times 2 m$ & 2,33 & 0,63 & - & - \\
\hline $4 \mathrm{~m} \times 3 \mathrm{~m}$ & 2,17 & 0,57 & 0,72 & - \\
\hline $4 \mathrm{~m} \times 4 \mathrm{~m}$ & 2,15 & 0,62 & 0,82 & 0,73 \\
\hline
\end{tabular}

A similaridade entre a composição florística encontrada, nos três espaçamentos analisados, foi acima de 70\%. Pode-se observar que houve grande homogeneidade na composição florística entre os três espaçamentos. A maior similaridade igual a $82 \%$ foi verificada entre o menor ( $4 \mathrm{~m} \times 2 \mathrm{~m}$ ) e o maior ( $4 \mathrm{~m} \times 4 \mathrm{~m}$ ) espaçamento.

No espaçamento $4 \mathrm{~m} \times 2 \mathrm{~m}$ foi observada maior diversidade de espécies comparativamente ao espaçamento $4 \mathrm{~m} \times 3 \mathrm{~m}$, cuja estimativa de riqueza foi maior do que a do primeiro. A maior diversidade verificada no espaçamento $4 \mathrm{~m} \times 2 \mathrm{~m}$ provavelmente foi influenciada pela maior abundância e sua equabilidade. Além disso, esses resultados sugerem também que o menor espaçamento utilizado, promovendo um ambiente de copa mais fechada na fase inicial do povoamento, pode ter favorecido a maior diversidade de espécies na área.

Da análise da curva de rarefação, reforçado pelo estimador Jackknife l, pode-se verificar que houve diferença na riqueza estimada, mostrando-se maior no espaçamento intermediário ( $4 \mathrm{~m} \times 3 \mathrm{~m}$ ). Porém, a riqueza nos três espaçamentos foi constituída por poucas espécies abundantes, sendo refletido nos baixos valores de equabilidade e diversidade. Sob o ponto de vista ecológico, para uma melhor preservação das espécies na área, é desejável que haja uma distribuição mais adequada dos indivíduos pelas espécies.

No banco de sementes de florestas sucessionais com 6, 17 e 30 anos, após exploração, na Amazônia Oriental, Araujo et al. (2001) encontraram índices de diversidade de Shannon de $2,23,1,90$ e 1,12 e de equabilidade de 0,52, 0,46 e 0,27. A diversidade encontrada nos espaçamentos $4 \mathrm{~m} \times 3 \mathrm{~m} \mathrm{e} 4 \mathrm{~m} \times 4 \mathrm{~m}$ foi levemente inferior à diversidade da floresta de 6 anos. Já no banco de sementes de um povoamento com cerca de 18 anos de Eucalyptus robusta Sm., no espaçamento $3 \mathrm{~m} \times 2 \mathrm{~m}$, os valores, apesar de serem baixos, foram um pouco superiores aos encontrados neste trabalho, com H'= 2,36 e J=0,70 (Nóbrega et al., 2009). Baixa diversidade, $\mathrm{H}^{\prime}=2,11$ e J= 0,67, foi encontrada também no trabalho de Braga et al. (2008), em um fragmento florestal com aproximadamente 40 anos de regeneração natural.

Os espaçamentos de plantio apresentaram entre si alta similaridade na composição florística, levando-se em consideração que valores do índice de Sørensen são mencionados como altos quando maiores que 0,5 (Felfili et al., 1992). Machado et al. (2013) explicam que alta similaridade de espécies entre diferentes ambientes pode ser causado pela semelhança histórica e da proximidade das áreas.

Das poucas espécies de árvores encontradas neste trabalho, Trema micrantha e Zanthoxylum rhoifolium, cuja densidade relativa foi muito baixa, com $0,13 \%$ e $0,09 \%$, são recomendadas por Martins (2013) para restauração florestal de Áreas de Preservação Permanente e Reserva Legal. Segundo esse mesmo autor, a primeira, pertence ao grupo ecológico das pioneiras, a segunda, das pioneiras e secundárias inicial, sendo que ambas possuem frutificação atrativa à fauna e são indicadas para áreas bem drenadas (Carvalho, 
2003). Outros autores também recomendaram Trema micrantha em projetos de restauração (Campos Filho \& Sartorelli, 2016; Stolarski et al., 2018). Espécies pioneiras crescem mais rápido oferecendo sombra necessária para a sobrevivência e o estabelecimento das secundárias tardias e clímax (Martins, 2013).

Para estimular a regeneração natural em áreas degradadas e aumentar a diversidade de espécies, Martins (2013) recomenda enriquecer e adensar o banco de sementes do solo com sementes de espécies arbóreas. Essa técnica também foi indicada por Gasparino et al. (2006), ao concluir que a revegetação das áreas estudadas, por apresentar quantidade reduzida de propágulos viáveis de espécies arbóreas, necessita de plantio de mudas ou semeadura direta de espécies arbóreas para acelerar a sucessão vegetal.

Um fator importante no presente trabalho de pesquisa foi a remoção dos tratos culturais de roçagem da vegetação espontânea, após aproximadamente quatro anos do estabelecimento do plantio de Tachigali vulgaris. Esse procedimento de manejo favoreceu a regeneração e o desenvolvimento de espécies no sub-bosque com sementes provenientes de fontes externas e do próprio banco de sementes do solo, conforme encontrado no trabalho de Narducci et al. (2020).

Um fato relevante é que diversas espécies, ausentes no banco de sementes do solo, encontravam-se presentes na regeneração natural do sub-bosque na área de plantio de Tachigali vulgaris, conforme verificado também por Narducci et al. (2020). Tais espécies são indicadas para recomposição de áreas alteradas, quais sejam: Aegiphila integrifolia (Jacq.) Moldenke, Tapirira guianensis Aubl. (Martins, 2013, 2014), Apuleia leiocarpa (Vogel) J.F.Macbr., Inga edulis Mart., Schefflera morototoni (Aubl.) Maguire et al. (Martins, 2013, 2014; Campos Filho \& Sartorelli, 2016), Bellucia grossularioides (L.) Triana, Caryocar villosum (Aubl.) Pers., Dipteryx odorata (Aubl.) Willd., Handroanthus serratifolius (Vahl) S.O. Grose, Jacaranda copaia (Aubl.) D. Don, Pouteria macrophylla (Lam.) Eyma, Schizolobium parahyba var. Amazonicum (Huber ex Ducke) Barneby (Campos Filho \& Sartorelli, 2016). Além dessas, há também a presença de Cecropia palmata Willd., uma das espécies com maior índice de valor de importância nos três espaçamentos (Narducci et al., 2020), que é potencialmente acumuladora de fósforo (Oliveira \& Carvalho, 2008). Essas evidências, mesmo com a predominância de herbáceas no banco de sementes em estudo, sugerem que não há necessidade de enriquecimento do banco de sementes do solo no processo de recuperação da área com finalidades econômica ou de paisagem.

\section{CONCLUSÕES}

A composição florística dos três espaçamentos de plantio de Tachigali vulgaris destacou- se pela presença e abundância de espécies caracterizadas pela forma de vida de erva.

A riqueza estimada foi diferente entre os espaçamentos, porém ocorreram poucas espécies abundantes influenciando na baixa diversidade e equabilidade florística.

Os três espaçamentos apresentaram elevada similaridade florística, com maior homogeneidade de espécies entre a maior $(4 \mathrm{~m} \times 2 \mathrm{~m})$ e menor $(4 \mathrm{~m} \times 4 \mathrm{~m})$ densidade de plantio.

Devido os três espaçamentos terem resultados semelhantes, ressalta-se que qualquer um pode ser viável para plantio com intuito de recompor e conservar o banco de sementes no solo. Neste caso, a escolha do espaçamento dependerá da viabilidade econômica de cada projeto de recuperação de áreas e da finalidade de uso dos indivíduos de Tachigali vulgaris plantados.

\section{REFERÊNCIAS}

Alvino-Rayol, F. O., \& Rayol, B. P. (2019). Efeito do fogo no banco de sementes do solo de sistemas agroflorestais, Pará, Brasil. Revista de Ciências Agroveterinárias, 18(4), 489-498. http://dx.doi.org/10.5965/223811711832019489.

Amaral, D. D., Vieira, I. C. G., Almeida, S. S., Salomão, R. P., Silva, A. S. L., \& Jardim, M. A. G. (2009). Checklist da flora arbórea de remanescentes florestais da região metropolitana de Belém e valor histórico dos fragmentos, Pará, Brasil. Boletim do Museu Paraense Emílio Goeldi, 4(3), 231-289. 
Araujo, M. M., Longhi, S. J., Barros, P. L. C., \& Brena, D. A. (2004). Caracterização da chuva de sementes, banco de sementes do solo e banco de plântulas em Floresta Estacional Decidual ripária Cachoeira do Sul, RS, Brasil. Scientia Forestalis, 66, 128-141.

Araujo, M. M., Oliveira, F. A., Vieira, I. C. G., Barros, P. L. C., \& Lima, C. A. T. (2001). Densidade e composição florística do banco de sementes do solo de florestas sucessionais na região do Baixo Rio Guamá, Amazônia Oriental. Scientia Forestalis, 59, 115-130.

Aumond, J. J. (2019). Restauração ambiental de sistemas complexos (303 p.). Curitiba: Appris.

Baider, C., Tabarelli, M., \& Mantovani, W. (1999). O banco de sementes de um trecho de floresta Atlântica Montana (São Paulo, Brasil). Revista Brasileira de Biologia, 59(2), 319-328. http://dx.doi.org/10.1590/S0034-71081999000200014.

Bloemer, J., Ternus, R. M., Cavalcante, J. A., Weiss, A. C., Folquini, P. S., \& Philippi, E. (2017). Manejo de plantas daninhas em função do arranjo populacional na cultura do milho. Tecnologia \& Ciência Agropecuária, 11(4), 27-32.

Bordon, N. G. (2012). Banco de sementes de uma floresta tropical madura e alterada por downburst na Amazônia Central (Dissertação de mestrado). Instituto Nacional de Pesquisas da Amazônia, Manaus.

Braga, A. J. T., Grifith, J. J., Paiva, H. N., \& Meira Neto, J. A. A. (2008). Composição do banco de sementes de uma floresta semidecidual secundária consideando o seu potencial de uso para recuperação ambiental. Revista Árvore, 32(6), 1089-1098. http://dx.doi.org/10.1590/S0100-67622008000600014.

Brandão, J. F. C., Martins, S. V., \& Brandão, I. J. (2016). Potencial de regeneração de uma área invadida por Pteridium aquilinum no Parque Nacional do Caparaó. Floresta, 46(4), 543-552. http://dx.doi.org/10.5380/rf.v46i4.41387.

Calegari, L., Martins, S. V., Campos, L. C., Silva, E., \& Gleriani, J. M. (2013). Avaliação do banco de sementes do solo para fins de restauração florestal em Carandaí, MG. Revista Árvore, 37(5), 871-880. http://dx.doi.org/10.1590/S0100-67622013000500009.

Campos Filho, E. M., \& Sartorelli, P. A. R. (2016). Guia de identificação de espécies-chave para a restauração florestal na região de Alto Teles Pires, Mato Grosso (248p.). São Paulo: The Nature Conservancy.

Carvalho, J. A., Justo Júnior, G., \& Figueiredo, G. C. (2015). Estimativa de colonização por plantas daninhas, com base no banco de sementes do solo, em cafezais com diferentes arranjos espaciais de cultivo. In Anais Eletrônicos da $7^{\circ}$ Jornada Científica e Tecnológica; $4^{\circ}$ Simpósio de Pós-graduação. Poços de Caldas: IFSULDEMINAS. Recuperado em 17 de julho de 2021, de https://portal.ifsuldeminas.edu.br/index.php/pesquisa-pos-graduacao-inovacao/103-anais-dajornada-cientifica/876-jornada-cientifica-volume-7-2015

Carvalho, P. E. R. (2003). Espécies florestais brasileiras: recomendações silviculturais, potencialidades e uso da madeira. Colombo: EMBRAPA/CNPAF. 640 p.

Carvalho, P. E. R. (2005). Taxi-Branco. Colombo: Embrapa Florestas.

Chao, A., Gotelli, N. J., Hsieh, T. C., Sander, E. L., Ma, K. H., Colwell, R. K., \& Ellison, A. M. (2014). Rarefaction and extrapolation with Hill numbers: a framework for sampling and estimation in species diversity studies. Ecological Monographs, 84(1), 45-67. http://dx.doi.org/10.1890/13-0133.1.

Costa, J. R., Fontes, J. R. A., \& Morais, R. R. (2013a). Banco de sementes do solo em áreas naturais e cultivos agrícolas. Manaus: Embrapa Amazônia Ocidental.

Costa, J. R., Mitja, D., \& Leal Filho, N. (2013b). Bancos de sementes do solo em pastagens na Amazônia Central. Pesquisa Florestal Brasileira, 33(74), 115-125. http://dx.doi.org/10.4336/2013.pfb.33.74.431.

Domingues, M. S., \& Bermann, C. (2012). O arco de desflorestamento na Amazônia: da pecuária á soja. Ambiente \& Sociedade, 15(2), 1-22. http://dx.doi.org/10.1590/S1414-753X2012000200002.

Favreto, R., \& Medeiros, R. B. (2006). Banco de sementes do sole em área agrícola sob diferentes sistemas de manejo estabelecida sobre campo natural. Revista Brasileira de Sementes, 28(2), 34-44. http://dx.doi.org/10.1590/S0101-31222006000200005.

Felfili, J. M., Silva Júnior, M. C. S., Rezende, A. V., Machado, J. W. B., Walter, B. M. T., Silva, P. E. N., \& Hay, J. D. (1992). Análise comparativa da florística e fitossociologia da vegetação arbórea do Cerrado sensu stricto na Chapada Pratinha, DF-Brasil. Acta Botanica Brasílica, 6(2), 27-46. http://dx.doi.org/10.1590/S0102-33061992000200003.

Flora do Brasil 2020 (2017). Jardim Botânico do Rio de Janeiro. Retrieved in 2017, January 30, from http://floradobrasil.jbrj.gov.br/

Fonseca, A., Amorim, L., Cardoso, D., Ribeirom, J., Ferreira, R., Kirchhoff, F., Monteiro, A., Santos, B., Ferreira, B., Souza Junior, C., \& Veríssimo, A. (2020). Boletim do desmatamento da Amazônia Legal (dezembro 2020) SAD. Belém: Imazon. 
Franco, B. K. S., Martins, S. V., Faria, P. C. L., \& Ribeiro, G. A. (2012). Densidade e composição florística do banco de sementes de um trecho de Floresta Estacional Semidecidual no Campus da Universidade Federal de Viçosa, Viçosa, MG. Revista Árvore, 36(3), 423-432. http://dx.doi.org/10.1590/S010067622012000300004.

Freitas, G. A., Vaz-de-Melo, A., Pereira, M. A. B., Andrade, C. A. O., Lucena, G. N., \& Silva, R. R. (2012). Influência do sombreamento na qualidade de mudas de Sclerolobium paniculatum Vogel para recuperação de área degradada. Journal of Biotechnology and Biodiversity, 3(3), 5-12. http://dx.doi.org/10.20873/jbb.uft.cemaf.v3n3.freitas.

Gasparino, D., Malavasi, U. C., Malavasi, M. M., \& Souza, I. (2006). Quantificação do banco de sementes sob diferentes usos do solo em área de domínio ciliar. Revista Árvore, 30(1), 1-9. http://dx.doi.org/10.1590/S0100-67622006000100001.

Gonçalves, A. R., Martins, R. C. C., Martins, I. S., \& Felfili, J. M. (2008). Banco de sementes do sub-bosque de Pinus spp. e Eucalyptus spp. Na Flona de Brasília. Cerne, 14(1), 23-32.

Gotelli, N. J., \& Colwell, R. K. (2011a). Estimating species richness. In A. E. Magurran \& B. J. McGill (Eds.), Biological diversity: frontiers in measurement and assessment (pp. 39-54). Oxford: Oxford University Press.

Gotelli, N. J., \& Ellison, A. M. (2011b). Princípios de estatística em ecologia (528 p.). Porto Alegre: Artmed.

Hsieh, T. C., Ma, K. H., \& Chao, A. (2020). iNEXT: Interpolation and Extrapolation for Species Diversity. $R$ package version 2.0.20.

Lau, A. V., \& Jardim, A. G. (2014). Composição e densidade do banco de sementes em uma floresta de várzea, ilha do Combu, Belém-Pa, Brasil. Biota Amazônia, 4(3), 5-14. http://dx.doi.org/10.18561/2179-5746/biotaamazonia.v4n3p5-14.

Macedo, A. C. (1993). Revegetação: matas ciliares e de proteção ambiental (24 p.). São Paulo: Fundação Florestal.

Machado, V. M., Santos, J. B., Pereira, I. M., Lara, R. O., Cabral, C. M., \& Amaral, C. S. (2013). Avaliação do Banco de Sementes de uma área em processo de recuperação em Cerrado Campestre. Planta Daninha, 31(2), 303-312. http://dx.doi.org/10.1590/S0100-83582013000200007.

Magurran, A. E. (2004). Measuring biological diversity (256 p.). Oxford: Blackwell Science.

Martins, S. V. (2013). Recuperação de áreas degradadas: ações em áreas de preservação permanente, voçorocas, taludes rodoviários e de mineração (270 p.). Viçosa: Aprenda Fácil.

Martins, S. V. (2014). Restauração florestal em áreas de preservação permanente e reserva legal. Viçosa: Aprenda Fácil.

Martins, S. V., Almeida, D. P., Fernandes, L. V., \& Ribeiro, T. M. (2008). Banco de sementes como indicador de restauração de uma área degradada por mineração de Caulim em Brás Pires, MG. Revista Árvore, 32(6), 1081-1088. http://dx.doi.org/10.1590/S0100-67622008000600013.

Miranda Neto, A., Kunz, S. H., Martins, S. V., Silva, K. A., \& Silva, D. A. (2010). Transposição do banco de sementes do solo como metodologia de restauração florestal de pastagem abandonada em Viçosa, MG. Revista Árvore, 34(6), 1035-1043. http://dx.doi.org/10.1590/S0100-67622010000600009.

Mochiutti, S., Melém Júnior, N. J., Farias Neto, J. T., \& Queiroz, J. A. L. (1999). Taxi-branco (Sclerolobium paniculatum Vogel): Leguminosa arbórea para recuperação de áreas degradadas e abandonadas pela agricultura migratória. Macapá: Embrapa Amapá.

Mochiutti, S., Queiroz, J. A. L., \& Melém-Júnior, N. J. (2006). Produção de serapilheira e retorno de nutrientes de um povoamento de taxi-branco e de uma floresta secundária no Amapá. Boletim de Pesquisa Florestal, 52, 3-20.

Monaco, L. M., Mesquita, R. C. G., \& Williamson, G. B. (2003). Banco de sementes de uma floresta secundária amazônica dominada por Vismia. Acta Amazonica, 33(1), 41-52. http://dx.doi.org/10.1590/1809-4392200331052.

Narducci, T. S., Yared, J. A. G., \& Brienza Junior, S. (2020). Regeneração natural do sub-bosque em plantios de Taxi-branco (Tachigali vulgaris L.F. Gomes da Silva \& H.C. Lima) sob diferentes espaçamentos na Amazônia Brasileira. Biota Amazônia, 10(3), 16-21. http://dx.doi.org/10.18561/2179-5746/biotaamazonia.v10n3p16-21.

Nóbrega, A. M. F., Valeri, S. V., Paula, R. C., Pavani, M. C. M. D., \& Silva, S. A. (2009). Banco de sementes de remanescentes naturais e de áreas reflorestadas em uma várzea do rio Mogi-Guaçu-SP. Revista Árvore, 33(3), 403-411. http://dx.doi.org/10.1590/S0100-67622009000300002.

Oliveira Júnior, R. S., Constantin, J., \& Inoue, M. H. (2011). Biologia e manejo de plantas daninhas (348 p.). Curitiba: Omnipax. 
Oliveira, P. C., \& Carvalho, C. J. R. (2008). Interações biofísicas em espécies arbóreas potencialmente acumuladoras de fósforo: diversidade de irradiância e de comportamento hídrico. Acta Amazonica, 38(3), 445-452. http://dx.doi.org/10.1590/S0044-59672008000300008.

Pará. Secretaria Executiva de Estado de Planejamento, Orçamento e Finanças - SEPOF. (2013). Estatística municipal: Dom Eliseu (53 p.). Recuperado em 20 de setembro de 2020, de http://iah.iec.pa.gov.br/iah/fulltext/georeferenciamento/domeliseu.pdf

Rayol, B. P., \& Alvino-Rayol, F. O. (2018). Chuva e banco de sementes no solo em agroecossistemas do Baixo Amazonas, Pará. Agrarian Academy, 5(10), 40-51. http://dx.doi.org/10.18677/Agrarian_Academy_2018B5.

Rodrigues, R. P., Gonçalves, D. A., Silva, A. R., Martins, W. B. R., Dionísio, L. F. S., \& Schwartz, G. (2020). Crescimento e mortalidade de Tachigali vulgaris L.G.Silva \& H.C.Lima em diferentes espaçamentos de plantio para a produção de biomassa. Scientia Forestalis, 48(128), e3269. http://dx.doi.org/10.18671/scifor.v48n128.01.

Roque, N., \& Bautista, H. P. (2008). Asteraceae: caracterização e morfologia floral (71 p.). Salvador: EDUFBA.

Seubert, R. C., Maçaneiro, J. P., Budag, J. J., Fenilli, T. A. B., \& Schorn, L. A. (2016). Banco de sementes do solo sob plantios de Eucalyptus grandis no Município de Brusque, Santa Catarina. Floresta, 46(2), 165-172. http://dx.doi.org/10.5380/rf.v46i2.38191.

Sousa, V. G. (2011). Comportamento silvicultural e dinâmica de serapilheira em plantios de duas espécies florestais na Amazônia oriental brasileira (Dissertação de mestrado). Instituto de Geociências, Universidade Federal do Pará, Museu Paraense Emílio Goeldi, Empresa Brasileira de Pesquisa Agropecuária, Belém.

Sousa, V. G., Brienza Junior, S., Barbosa, M. G., \& Martorano, L. M. (2016). Taxi-branco (Tachigali vulgaris L.F. Gomes da Silva \& H.C. Lima): botânica, ecologia e silvicultura. Belém: Embrapa Amazônia Oriental.

Souza, C. R., Lima, R. M. B., Azevedo, C. P., \& Rossi, L. M. B. (2004). Taxi-branco (Sclerolobium paniculatum Vogel). Manaus: Embrapa Amazônia Ocidental.

Stolarski, O. C., Gorenstein, M. R., Lubke, M., Lubke, L., O'Connor, P. H. P., \& Bechara, F. C. (2018). Trema micrantha (L.) Blume. In Plantations for ecological restoration: early development in the Brazilian subtropical forest. Ciência Florestal, 28(3), 1217-1229. http://dx.doi.org/10.5902/1980509833351.

Tropicos (2017). Missouri Botanical Garden. Retrieved in 2017, January 30, from https://tropicos.org

Villarreal, H., Álvarez, M., Córdoba, S., Escobar, F., Fagua, G., Gast, F., Mendoza, H., Ospina, M., \& Umaña, A. M. (2004). Manual de métodos para el desarrollo de inventarios de biodiversidade (236 p.). Bogotá: Instituto de Investigación de Recursos Biológicos Alexander von Humboldt.

Zappi, D. A., Gastauer, M., Ramos, S., Nunes, S., Caldeira, C. F., Souza-Filho, P. W., Guimarães, T., Giannini, T. C., Viana, P. L., Lovo, J., Mota, N. F. O., \& Siqueira, J. O. (2018). Plantas nativas para recuperação de áreas de mineração em Carajás (286p.). Belém: Instituto Tecnológico Vale.

Contribuição dos Autores: TSN: conceituação, curadoria de dados, análise formal, investigação, metodologia, validação, visualização, escrita - primeira redação, escrita - revisão e edição; JAGY: conceituação, metodologia, validação visualização, supervisão, escrita - primeira redação, escrita - revisão e edição; SBJ: conceituação, metodologia, visualização, supervisão, administração do projeto, recursos, escrita - revisão e edição. 